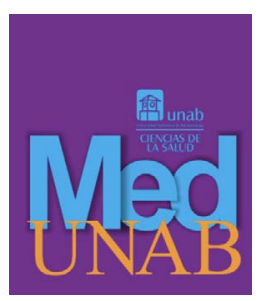

REVISTA DE LA FACULTAD

DE CIENCIAS DE LA SALUD

Vol. 24(1): 6-8, april -july 2021

i-SSN 0123-7047

-ISSN 2382-4603

DOI: https://doi.org/10.29375/01237047.4019

\title{
Medical education and research during the COVID-19 pandemic: How to meet the challenges posed
}

\author{
Educación e investigación médica durante la pandemia de COVID-19: cómo \\ enfrentar los desafíos planteados
}

\section{Educação médica e pesquisa durante a pandemia COVID-19: como enfrentar os desafios colocados}

The COVID-19 pandemic has prompted numerous challenges for the field of education, particularly medical education and health-related research. However, these obstacles are also an opportunity to advance this academic-medical sphere with the increased introduction of information technologies and the creation of strategies that focus on quality improvement. Previous research has highlighted the importance of adapting both clinical practice and medical education within the context of the pandemic (1). As COVID-19 continues to surge across the globe, the gaps in medical education and research grow and they must be met urgently with a creative approach toward making the available technology accessible and relevant.

Efforts to improve the medical curriculum and scientific research within the context of this pandemic are crucial at this difficult time. This is especially true in environments that generally require assembling, such as in academic meetings and other research-related activities. Many clinicians, students and researchers do not consider it feasible to follow the rigorous procedures set by international guidelines and local regulations (2). In such instances, health care professionals have mostly prioritized clinical activities in order to maintain social distancing and limiting any extraneous activities (1). Accordingly, learning sessions have been adjusted to virtual settings and proposals for research procedures to be streamlined and paperwork minimized while continuing to follow the set protocols have been set forth. These changes have been implemented in accordance with Good Clinical Practice (GCP) standards, in order to produce reliable data while also ensuring participant safety (1). 
Acknowledging the impact of the ongoing COVID-19 outbreak on medical education, the Liaison Committee on Medical Education has updated resources on safety and education to help medical schools uphold their core competencies and maintain their accreditation (3). Furthermore, different organizations related to clinical research, such as the European Medicines Agency (EMA) and GCP, among others, have laid out new guidelines on managing clinical trials during the COVID-19 pandemic. They address the needs of the global crisis by promoting greater flexibility and procedural simplifications while preserving the quality of clinical trials and guarding the safety of study participants (4).

The challenges posed by this "new normality," as the pandemic-related restrictive environment has been dubbed, involve adopting new and ingenious methodologies for teaching, learning and researching with a flexible approach to facilitate healthcare provision and clinical education in a way that is safe for patients, students and health professionals. Optimizing online clinical didactic sessions, expanding the use of virtual clinical cases and strengthening the tele-health environment are part of the suggestions that have been made in recent publications related to medical education during the COVID-19 pandemic (5). While these steps are important, they are not sufficient. Software engineers must design machine learning systems to work together with applications used to record how students and clinicians learn best and where their weaknesses lie, in order to continuously improve. Moreover, the programs used need to encourage greater conversation among members of the medical field, both within institutions and with other medical centers, even in remote corners of the world.

Likewise, telemedicine needs to be enhanced for patients to better prepare for these visits and have the tools to measure various biomarkers, when possible. Moreover, clinicians need to be provided with specific training on how to conduct these visits most efficiently and fruitfully. On top of that, new guidelines must be set to help screen medical students and residents in training for signs of burn-out and issues related to mental health, and to implement individualized stressmanagement programs to ensure their well-being and safety, especially during these isolating times (1).

Recently, a systematic review evaluated the evidence concerning medical educational developments in response to the pandemic (6). The study synthetized the results of 49 studies carried out in different countries. Some highlights include the implementation of an online learning approach to deliver seminars, debates and run simulation and clinical skill sessions; the use of supervised phone or video consultations to support clinical learning without requiring physical presence within the clinical settings; and the development of virtual ward rounds using an iPad on wheels in order to interact in real time with patients and physicians (6). The review describes the positive aspects of remote learning as identified by the studies including improvements in efficiency, flexibility and communication among other benefits. Nonetheless, the numerous challenges related to training in the use of online platforms and novel technologies, increasing access to WiFi and bandwidth and follow-up of the different developments in medical education must be addressed (6). The success of these measures in adapting to the urgent medical needs of the current global crisis will determine their long-term impact, and ultimately, shape how we provide medical care and train rising clinicians moving forward.

In summary, improving clinical practice, medical education and health-related research requires the commitment of the different players within the health system. The pandemic offers an opportunity for both students and teachers to analyze and document the challenges they are facing and brainstorm creative solutions that can be provided by the available technology. The answers that arise will only strengthen the medical curriculum and the way we conduct research processes. They will foster greater academic collaboration across career stages, specialties, and global borders, ultimately ensuring improved validation and reliability in published research (7). While the duration of the COVID-19 crisis remains uncertain, what is clear is that the actions taken in light of the challenges posed by this pandemic will significantly transform the way we understand and teach medicine.

iD Yuli Guzman-Prado Medical Doctor, Master in Epidemiology, Master in Healthcare Management. Member of MedUNAB journal (editorial board).

iD Jennifer Ben Shimol Medical Doctor, Rheumatologist. Department of Medicine, E. Wolfson Medical Center, Israel. Sackler Faculty of Medicine, Tel Aviv University, Tel Aviv, Israel. 


\section{References}

1. Guzman-Prado Y, Vita R, Ben Shimol J. Developing a toolkit to navigate clinical, educational and research practice during the COVID-19 pandemic. International Journal of Clinical Practice. 2020. DOI: https://doi. org/10.1111/ijcp.13676

2. Maggioni A, Rapezzi C, Tavazzi L, Ferrari R. Key words to be adopted for COVID-19 research: A return to simple, large, randomized trials. European Heart Journal. 2020. DOI: https://doi-org/10.1093/eurheartj/ ehaa322

3. COVID-19 updates and resources. Liaison Committee on Medical Education. Updated March 25, 2020. Available online at: https://cme.org/covid-19/ (accessed October 22, 2020).

4. Guidance on the management of clinical trials during the COVID-19 (coronavirus) pandemic. European Medicines Agency. Available online at: https:// ec.europa.eu/health/sites/health/files/files/eudralex/vol10/guidanceclinicaltrials covid19 en.pdf (accessed October 22, 2020).

5. Rose S. Medical Student Education in the Time of COVID-19. JAMA. 2020;323(21):2131-2132. DOI: http://doi.org/10.1001/jama.2020.5227

6. Gordon M, Patricio M, Horne L, Muston A, Alston SR, Pammi M, Thammasitboon S, Park S, Pawlikowska T, Rees EL, Doyle AJ. Developments in medical education in response to the COVID-19 pandemic: a rapid BEME systematic review: BEME Guide No. 63. Medical teacher. 2020;42(11):1202-15. DOI: https:// doi.org/10.1080/0142159X.2020.1807484

7. Guzman-Prado Y. Retraction of Studies on Potential Drug Therapies for COVID-19: A Call for Reliability and Scientific Integrity. American Journal of Cardiology. 2020;132:173. DOI: https://doi. org/10.1016/j.amjcard.2020.06.061 